\title{
Pour un dialogue interculturel: évolution sémantique du Nom de Marque Kärcher dans le discours
}

\author{
For an intercultural dialogue: semantic evolution \\ of the Kärcher brand name in discourse
}

\author{
Michela TonTI \\ Alma Mater Studiorum Università di Bologna \\ michela.tonti2@unibo.it
}

\begin{abstract}
The aim of our work is twofold: the exploration of corpora combined with the search for an innovative modality for refining intercultural competence. For this purpose, we use the brand name Kärcher, which for many years has largely departed from its original meaning as a high-pressure water cleaner by acquiring semantic weight and discursive versatility through discourse, as it blends with other semantic-syntactic combinatorics for the linguistic needs of speakers.

In a first step, we will demonstrate that the name Kärcher, which by antonomasia was associated with "scum" at its origins, evolves in discourses and affects other spheres. Namely, native French-speaking writers or not, appropriate the Brand Name Kärcher and transform it as they please by applying it to the most distant domains compared to that of cleanliness in the literal and figurative sense of the term. Finally, interculturality as a "dynamic between" escapes the seemingly fixed side of culture and manifests itself as a dynamic between different domains.
\end{abstract}

\begin{abstract}
Resumen
El objetivo de nuestro trabajo es doble: la exploración de corpus combinada con la búsqueda de una modalidad innovadora para perfeccionar la competencia intercultural. Para ello, utilizamos el nombre de marca (NdM) Kärcher, que durante muchos años se ha desviado en gran medida de su significado original de limpiador de agua a alta presión, adquiriendo grosor semántico y versatilidad discursiva a través del discurso, al casarse con otras combinatorias semántico-sintácticas para las necesidades lingüísticas de los hablantes.

En primer lugar, demostraremos que el nombre Kärcher, que por antonomasia se asociaba a "escoria" en sus orígenes, evoluciona en los discursos y toma vuelos insospechados que afectan a otras esferas. A saber, los escritores francófonos nativos o no se apropian del NdM Kärcher y lo transforman a su antojo para aplicarlo a los dominios más lejanos en comparación con el de la limpieza en el sentido literal y figurado del término. Por último, la interculturalidad como "dinámica entre" escapa al lado aparentemente fijo de la cultura y se manifiesta como una dinámica entre diferentes ámbitos.
\end{abstract}


Anales de Filología Francesa, n. ${ }^{\circ}$ 29, 2021

POUR UN DIALOGUE INTERCULTUREL: ÉVOLUTION SÉMANTIQUE DU NOM DE MARQUE KÄRCHER...

\section{Keywords}

Corpus linguistics, brand names, cross-cultural dimension, lexiculture, discourse referent.

\section{Palabras clave}

lingüística de corpus, nombres de marca, dimensión transcultural, lexicultura, referente del discurso.

\section{Introduction}

En didactique des langues se pose la question de l'accès à la langue, la variation formant une partie intégrale, nécessaire de la langue à enseigner (Sinclair, 2004). Or, une conception de la langue qui fait une place à des usages, à des tendances et à des faits observés, présente un certain nombre de problèmes pour la didactique des langues (Conrad, 2010): la "référence" est désormais un ensemble d'informations qui émergent des données (cité par Boulton \& Tyne (2014: 137). De nos jours, les étudiant.e.s ont connu la généralisation du web, l'utilisation de Google comme outil de vérification linguistique, le développement des dictionnaires et des bases de données terminologiques en ligne.

L'objectif de notre travail porte sur une double exigence. D'un côté, nous valorisons l'exploration de corpus combinée à la quête d'une modalité innovante pour travailler la dimension interculturelle; de l'autre, nous ciblons la recherche des collocations d'usage à propos d'unités lexicales simples ou multilexémiques en perspective culturelle. Ce travail s'inscrit dans le cadre d'une formation universitaire en médiation culturelle. Pour ce faire et dans le sillage de Robert Galisson (Galisson, 1991; Galisson \& André, 1998), nous analysons le nom de marque (désormais NdM) Kärcher qui s'est largement détaché de son sens initial de nettoyeur à eau à haute pression depuis de longues années, en acquérant à travers les discours des médias: épaisseur sémantique et polyvalence discursive, car il se marie avec d'autres combinatoires sémantico-syntaxiques pour les besoins langagiers des parlants (Tonti, 2019; Tonti, 2020).

Comment au départ une lexie qui servait une cause commerciale s'est-elle retrouvée à servir de sas de reconnaissance, de transformation et d'évolution des idées, des concepts afférant aussi bien à la sphère du concret que de l'abstrait? Dans quelle mesure garantissait-elle ainsi des branchements interculturels insoupçonnés auparavant? Comment la lexie Kärcher et ses dérivés parviennent-ils à colorier une large palette des grands secteurs d'activité allant du domaine politique, socio-économique, écologique, du droit jusqu'à la vie quotidienne et ludique? 
Anales de Filología Francesa, n. ${ }^{\circ}$ 29, 2021

MICHELA TONTI

\section{Cadre théorique}

\subsection{Pour une typologisation du concept de l'interculturel}

Dans un premier temps, nous nous sommes tournée vers le cadre théorique afférant au concept de l'interculturel tracé par Jacques Demorgon qui souligne que

on associe presque toujours le champ notionnel de l'interculturel aux relations entre personnes, appartenant à des pays de culture différente et, par extension, aux relations entre ces pays [...]. La relation entre les cultures des personnes et celle des pays pose la nécessité d'éviter deux erreurs: penser les pays comme déterminant simplement la culture des personnes ou penser les personnes comme plus autonomes qu'elles ne le sont. (2003: 53)

Cette première observation nous amène à réfléchir sur le fait que selon l'auteur, l'interculturel se manifeste à travers ses acteurs qui s'investissent par une dynamique de groupe aussi bien dans les grands domaines de la société comme la politique, la religion, l'économie que dans l'informationnel (2003: 62). Parmi les visages de l'informationnel, Demorgon inclut le domaine technique, écologique, médiatique, ludique, entre autres. L'interculturel est ainsi entretenu par les différentes catégories d'acteurs entraînant leur "société singulière à se maintenir ou à se modifier" (2003: 63).

Lors de nos recherches, nous nous sommes ensuite intéressée aux travaux des chercheur.e.s comme Lemoine, qui a orienté ses études sur l'interculturel au champ de l'éducation et de la linguistique. Elle a notamment interrogé l'interculturel en tant que "construit social sans cesse réactualisé par les personnes dans les relations, dans les expériences" (2018: 79). Il nous semble de toute évidence important de retenir cette définition qui prend en compte le potentiel évolutif des personnes et de la langue à la fois. Puisqu'on ne peut pas connaître autrui, sa manière de s'exprimer sans échanger avec lui, nous nous focalisons sur le préfixe inter de la notion de l'interculturel, sans négliger la culture, cependant.

Nous appréhendons ici l'interculturel comme une dynamique qui s'instaure entre personnes, pas forcément des "catégories", mais des êtres sociaux qui partagent une mémoire collective, celle d'un mot-événement lié à la lexie Kärcher et à une affaire politico-sociale qui a marqué les esprits des francophones de France.

Pour l'étude présentée dans cet article, nous nous situons dans le contexte de la blogosphère et des discours qui se tissent dans cet espace numérique largement décloisonné pour appréhender l'interactivité créatrice mobilisée par les écrilecteurs de blogs, pour la plupart personnels. Il s'agit d'une interactivité créatrice "entre" plusieurs domaines de l'activité humaine projetée par la lexie Kärcher qui sera analysée dans une perspective lexicographique, plutôt que terminologique. Kärcher ${ }^{1}$ est, en effet, un nom de marque, un terme déposé régi

1 Nous continuons d'utiliser le signe Kärcher en italique parce qu'il préserve son droit de marque bien qu'il circule énormément comme lexie de la langue commune. 
par le droit de la propriété intellectuelle mais ayant intégré le stock lexiculturel des francophones de France après les événements de $2005^{2}$. Il s'affranchit donc de sa gangue juridique pour prendre des envols insoupçonnés dans le français parlé ${ }^{3}$ dit "ordinaire" ${ }^{4}$ comprenant le discours de la presse hexagonale et de l'Assemblée Nationale.

\section{Corpus, méthodologie d'investigation}

À partir de deux corpus: Araneum (2015 1,2 milliard de tokens) et frTenTen (2017 10 milliards de tokens environ), nous établissons une exploration corpus-based par approche diachronique du NdM Kärcher qui n'a cessé de faire l'objet de réélaborations en discours avec des pointes de créativité lexicale de la part de scripteurs de blogs personnels, blogs réservés aux abonnés de la presse hexagonale et acteurs relevant de sites institutionnels.

Araneum est un corpus diversifié en genres textuels et largement échantillonné, qui fournit des indications permettant de donner aux phénomènes une représentation fondée sur un système d'usages, de pratiques discursives réelles. De surcroît, frTenTen a l'avantage d'exploiter une plus grande variété de typologies textuelles, comprenant également des textes littéraires en plus des formes de discours moins surveillées, comme les blogs et les forums. L'objet d'étude de notre problématique qui est de type représentationnel et interprétatif est défini à travers des hypothèses de représentations socio-discursives dont on suppose qu'elles sont dominantes à un moment donné (en l'occurrence l'année 2015 et l'année 2017 pour notre étude) et qu'elles caractérisent les locuteurs en fonction de leur groupe social d'appartenance et de surcroît d'appartenance politique, idéologique et sociale. L'éparpillement typique de l'Internet est contrecarré par le fait que les corpus constituent des supports conçus sur mesure.

L'authenticité des données réunies est assurée parce que la langue des textes confluant dans les corpus reflète celle des contextes d'usage des blogs personnels. L'objectif du blog est de faire partager, diffuser, faire une place à ce que l'on aime, notamment ses NdM préférés, auprès des personnes, mais aussi d'organiser, de hiérarchiser et de communiquer sa propre vision des objets de discussion, de débat, des circonstances et des idées du monde dans une perspective interculturelle. Autrement dit, les écrilecteurs s'expriment et mobilisent une part de subjectivité. L'interaction peut se construire dans l'accord, dans le désaccord ou encore être régie par des négociations entre je et la collectivité des écrilecteurs.

2 Allusion à la phrase polémique du candidat à la primaire de la droite et du centre en 2005, lors d'un déplacement à la Courneuve, qui voulait "nettoyer au Kärcher" la "racaille".

3 Nous renvoyons à la question du "français parlé” développée par des linguistes comme BENZITOUN, Christophe \& Gagnon, Roxane. 2020. "Le français parlé comme objet d'enseignement? Regards croisés d'une didacticienne et d'un linguiste" in Revue des HEP, numéro thématique La circulation des savoirs de la recherche en didactique(s) entre les espaces de l'enseignement, de la recherche et de la formation, $\mathrm{n}^{\circ} 26,37-51$.

4 Blanche-Benveniste, Claire. 2010. Approches de la langue parlée en français. Paris. Ophrys. 


\subsection{Quelles données pour l'exploration de la perspective interculturelle?}

$\mathrm{Au}$ vu de notre perspective, il nous faut préciser que lorsque nous disposons de données issues des discours numériques, il faut "repenser la conception de l'énonciation et le schéma persistant de la situation d'énonciation basé sur les quatre paramètres locuteur-interlocuteur-temps-lieu" (Paveau, 2017: 24-25). Les énoncés numériques possèdent en effet des caractéristiques particulières qui modifient en partie les grilles d'analyse traditionnelles comme l'investigabilité des textes: la mémoire du texte peut se perdre et donc avec lui ses métadonnées et notamment sa date de parution rendant parfois difficile d'observer en diachronie l'évolution de certains usages. Les textes sont parfois susceptibles d'être effacés de leur emplacement d'origine, pour la plupart des blogs personnels, ce qui s'avère partiellement un problème pour nous aussi, notamment pour le corpus Araneum.

Le cadre du monde connecté nous amène également à porter notre réflexion sur les extensions de domaine Internet: nous allons comprendre si une tendance de nationalisation culturelle du Web demeure dans le choix d'une zone de nommage en ".fr", plutôt que par un niveau générique comme ".net”, “.com”, “.org”, pour aller au-delà de l'idée de l'Internet comme un espace virtuel déconnecté de la géographie. Les extensions en ".fr", ".be", ".ca" ou ".ch" seraient-elles autant de marqueurs nationaux pour ce $N d M$ et donc seraient-elles porteuses d'enjeux culturels et interculturels? Les effets supposés entièrement globalisants de l'Internet sont-ils aussi constatables pour le NdM choisi?

L'article de Djordje Sredanovic, Les industries culturelles et la permanence du national à l'ère de l'Internet (2014) insiste sur la permanence de marqueurs nationaux d'ordre linguistico-culturel pour les produits culturels ${ }^{5}$ circulant dans le Net tout en étant dépendants des règlementations étatiques. Il signale même des tendances à une certaine renationalisation en relation avec l'individualisation et la personnalisation des pratiques culturelles et communicationnelles. Nous pouvons d'ailleurs inscrire les blogs dans ces formes d'individualisation et de personnalisation communicationnelle, et nous rappelons que lorsque nous avons sélectionné nos discours sur les $N d M$, ce sont davantage des contextes issus de blogs personnels que nous avons recensés; en revanche, les blogs de la presse relèvent davantage d'un discours plus contrôlé à juste titre car elle se doit d'être rigoureuse à l'égard des titulaires de la marque qui veillent au correct emploi de leur signe linguistique et du précieux champ sémantique qu'ils réservent de droit $^{6}$. Nous nous sommes interrogée sur les modalités d'obtention d'une

5 Sredanovic désigne la musique, les films, les livres, les fictions télévisées comme produits culturels. Nous considérons qu'une marque et son nom sont un produit culturel, en plus d'un produit défini selon les règles du marketing, du droit et les réflexions de la sémiotique.

6 Règlement (CE) No 207/2009 du Conseil, Article 10: Reproduction de la marque communautaire dans les dictionnaires: "Si la reproduction d'une marque communautaire dans un dictionnaire, une encyclopédie ou un ouvrage à consulter similaire donne l'impression qu'elle constitue le terme générique des biens ou services pour lesquels la marque est enregistrée, l'éditeur veille, sur demande du titulaire de la marque communautaire, à ce que la reproduction de la marque communautaire soit, au plus tard lors de l'édition suivante de l'ouvrage, accompagnée de l'indication qu'il s'agit d'une marque enregistrée". 
extension générique d'une part, et géographique ou nationale d'autre part, pour un utilisateur lambda; pour ce faire, nous avons contacté $1^{\prime} \mathrm{AFNIC}^{7}$. Conformément à la loi du 22 mars 2011, les enregistrements de noms de domaine en ".fr" ont été ouverts aux personnes physiques et morales résidant sur le territoire de l'Union Européenne. Il serait donc logique de croire que n'importe quel site ouvert avec extension de domaine en ".fr" et depuis n'importe quel pays de l'Union Européenne puisse être considéré comme un espace web national.

Nous nous sommes tournée vers nos corpus respectifs pour mesurer la diffusion par zone de nommage des lexies comportant l'unité "kärch" ou débutant par celle-ci. Cela exige une recherche aussi bien par lettre capitale que par lettre minuscule pour le corpus Araneum, à savoir, une recherche séparée. La même fouille outillée a été lancée par le biais de Sketch Engine au sein du corpus frTenTen. Il en découle les résultats suivants:

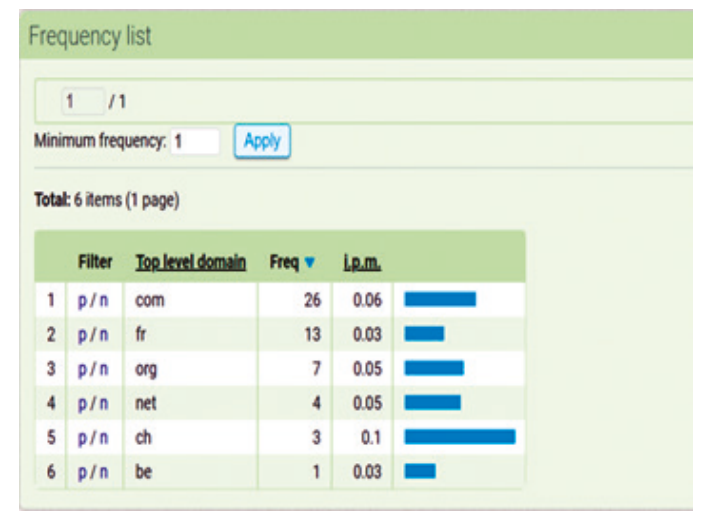

Figure 1. Liste des fréquences des lexies comprenant l'unité "kärch" classées par extension de domaine issue de Araneum.

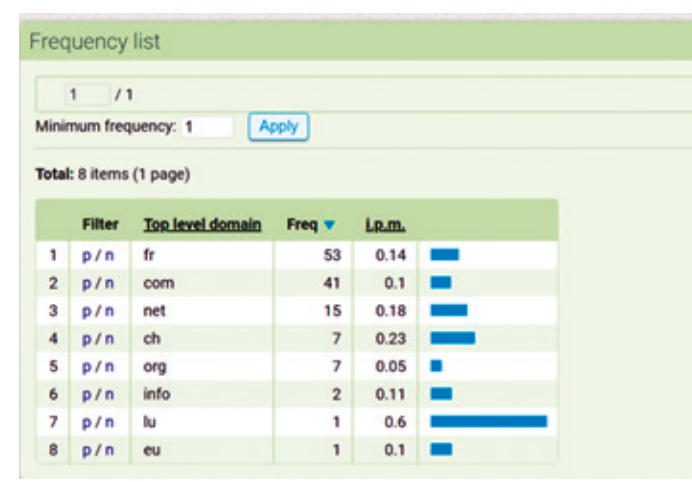

Figure 2. Liste des fréquences des lexies commençant par l'unité "Kärch" classées par extension de domaine issue de Araneum.

7 Association française pour le nommage Internet en coopération. 


\begin{tabular}{|c|c|c|c|c|}
\hline & & Top-level domain (e.g com) & Frequency $\downarrow$ & Relative in text type? \\
\hline 1 & 口 & if & 121 & 0.04 \\
\hline 2 & 口 & com & 51 & 0.04 \\
\hline 3 & $\square$ & org & 44 & 0.02 \\
\hline 4 & $\square$ & net & 16 & 0.06 \\
\hline 5 & $\square$ & info & 3 & 0.04 \\
\hline 6 & $\square$ & am & 1 & 2.15 \\
\hline 7 & 口 & eu & 1 & 0.02 \\
\hline
\end{tabular}

Figure 3. Liste des fréquences des lexies comprenant l'unité "kärch" classées par extension de domaine issue de frTenTen.

À l'issue de l'examen des résultats, il ressort que la zone de nommage nationale ".fr" dépasse largement, en termes de fréquence, l'extension générique “.com” aussi bien au sein des concordances issues de Araneum (Figure 2.) que de frTenTen (Figure 3.). Il n'est pas inutile de rappeler que les extensions de domaine nationale des pays d'expression française: ".be", “.ch", “.ca" sont inexistantes au sein de la liste des fréquences issue de frTenTen et fort peu représentées dans Araneum. Cela montre qu'un produit culturel comme un $N d M$ et davantage son signe linguistique peuvent avoir un enjeu culturel et interculturel certain mais qui traverse les esprits des bloggeurs, de leurs suiveurs et de la communauté toute de la Toile à condition qu'un lien fort d'association idéologique, sociale, politique s'installe avec ce nom événement à ses origines. La lexie "Kärcher" s'est notamment fait une place dans l'esprit des internautes francophones dont le choix de la zone de nommage nationale ".fr" pour leur site ne semblerait pas innocent. Ils semblent attribuer un poids considérable à l'événement déclencheur et à la polémique sociale qui a été tissée autour de lui, ce qui ne ferait pas l'objet d'un partage collectif pour les internautes ayant choisi d'autres extensions nationales relevant de l'espace francophone (“.be”, “.ch”, “.ca”). Finalement, notre première enquête semble permettre d'envisager que le choix d'une extension de domaine national n'est pas fortuite, en vertu, probablement, d'un enjeu tout d'abord identitaire.

\section{De l'antonomase à la dynamique entre domaines différents}

L'usage du nom (de la dénomination, en fait), grâce au pouvoir catégorisant de celuici, confère à l'événement son identité et son individualité: "Dès lors qu'un événement a été identifié sous une description, une dénomination spécifiant la typologie événementielle [...], son explication et son interprétation sont orientées et délimitées par la teneur sémantique des termes utilisés par cette description" (Neveu \& Quéré, 1996: 15) cité par Cislaru (2013). Par ailleurs, il faut souligner que 
tout ce qui arrive ne fait pas événement, mais seulement ce qui surprend notre attente, ce qui est intéressant, ce qui est important; par-là l'ordre des choses est vu du point de vue de notre préoccupation, de notre souci, donc, sous un horizon d'historicité qu'il faudra considérer plus loin. [...] L'événement c'est le nouveau par rapport à l'ordre institué. (Ricoeur, 1991: 43)

Le nouveau, par rapport à l'ordre institué, signifié par le NdM Kärcher correspond à la nouvelle vie de celui-ci lorsqu'en 2005, le Ministre de l'Intérieur de l'époque, N. Sarkozy promet de "nettoyer au karcher" la cité des 4000 dans le département 93 de la Seine-SaintDenis suite à un règlement de comptes ayant fait une victime.

Nous puisons dans nos corpus respectifs: Araneum et frTenTen pour constituer notre base empirique de données. L'étendue des dérivations et des néoformations témoigne du dynamisme et de l'innovation morpho-sémantique du $N d M$.

\begin{tabular}{|c|c|c|c|c|}
\hline \multicolumn{5}{|c|}{ Frequency list } \\
\hline \multicolumn{5}{|c|}{$1 / 1$} \\
\hline \multicolumn{3}{|c|}{ Minimum frequency: 1} & Apply & \\
\hline \multicolumn{5}{|c|}{ Total: 5 items (1 page) } \\
\hline & Filter & word & Ereq & \\
\hline 1 & $p / n$ & kărcher & 50 & E \\
\hline 2 & $p / n$ & kärcheriser & 1 & I \\
\hline 3 & $p / n$ & kärcherise & 1 & $\mathbf{I}$ \\
\hline 4 & $p / n$ & kärchers & 1 & I \\
\hline 5 & $p / n$ & kărcherisation & 1 & $\mathbf{I}$ \\
\hline
\end{tabular}

Figure 4. Liste des fréquences de la lexie Kärcher et de ses dérivés issues de Araneum.

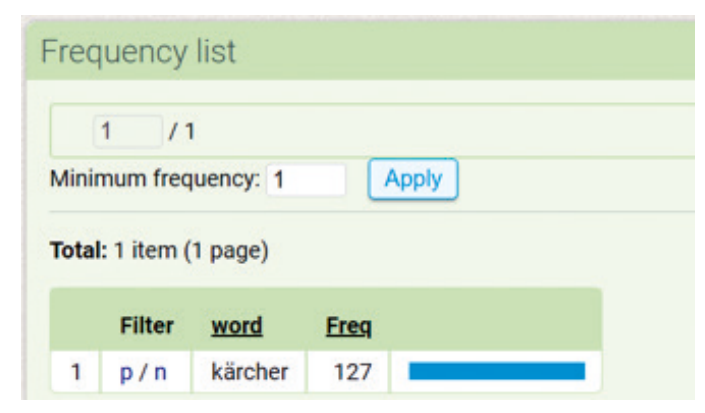

Figure 5. Occurrences de la lexie Kärcher issues de Araneum. 

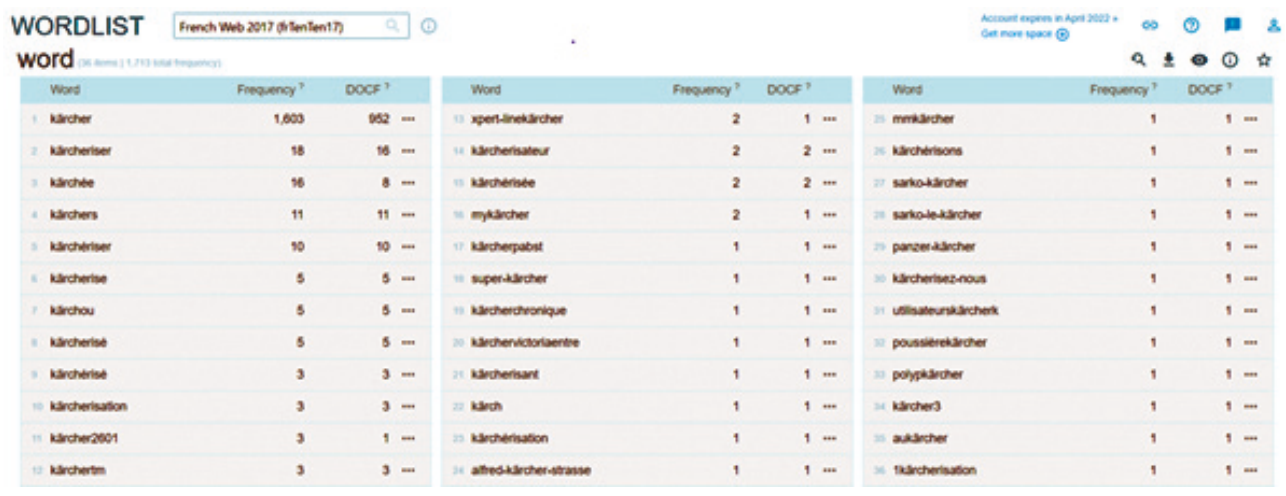

Figure 6. Liste des fréquences de la lexie Kärcher et de ses dérivés issues de frTenTen.

À la lecture des tableaux 4. et 6., pour laquelle nous nous appuyons sur la grille des matrices lexicogéniques conçue par Pruvost et Sablayrolles (2016 [2003]), il est indéniable que les formes néologiques répertoriées sont principalement le fruit du mécanisme morphosémantique de la suffixation: -ation est l'une des favorites pour signifier des transformations. Aucun suffixe ne permet de créer des verbes en dehors de -iser, -er étant le seul morphème productif ("kärcheriser", "kärchériser"). En revanche, parmi les formations à valeur adjectivale, notamment avec l'adoption de marques flexionnelles de la nouvelle catégorie, on détecte: "kärché", en guise d'exemple: "Vous allez encore vous faire de l'argent! rugit Me Szpiner $^{8}$ faisant allusion aux 500.000 exemplaires vendus du fameux numéro de Charlie ${ }^{9}$ sur les caricatures mais aussi, sans doute, aux 200.000 exemplaires vendus de la Face kärchée ..." (frTenTen, actuabd.com, 21 février 2007).

La composition simple dont le schéma syntaxique le plus productif demeure celui qui est composé de NOM + NOM prévoyant un trait d'union ou bien une soudure des deux lexies est également présente dans nos lexies: "panzer-kärcher", "kärcherpabst” (sic).

Ces néoformations ${ }^{10}$ se situent sur le plan du "méta-événement" ou discours sur l'événement, puisqu'elles deviennent événement à leur tour. L'événement par le biais de la comparaison ou de l'emploi d'un nom d'événement met en place des séries d'événements, en guise d'exemple: "kärcherpabst"11 (sic). L"“analogie intrinsèque" (Cislaru, 2013) est ba-

8 Avocat et homme politique français qui a défendu plusieurs personnalités impliquées dans des affaires politico-financières (source Wikipedia).

9 Nous rappelons que le fameux numéro de Charlie fait allusion à l'affaire des caricatures de douze dessinateurs parues le 30 septembre 2005 dans un quotidien danois donnant lieu à une controverse sur la liberté de presse. L'affaire prend une ampleur internationale, avec des manifestations dans certains pays, tantôt pacifiques tantôt violentes. Charlie Hebdo reprend ces "caricatures de Mahomet” quelques mois plus tard. Le contrôle de la production graphique est devenu un enjeu d'une extrême férocité qui touche aussi bien la politique que la religion.

10 À cet effet, nous renvoyons notre lecteur à Tonti (2020) pour de plus amples approfondissements à l'égard de la créativité lexicale et de l'évolution d'un $N d M$ du point de vue néologique et notamment à l'épreuve des mécanismes morpho-sémantique, nous signalons le passage suivant: Tonti, 2020: 139-153.

11 Nous avons reproduit l'erreur du scripteur du billet recueilli dans frTenTen. L'orthographe allemande est papst. 
sée sur les effets de l'événement à ses origines, à savoir, pendant le pontificat de Benoît XVI, l'Église est agitée par la révélation d'abus sexuels perpétrés surtout dans les décennies précédentes et contre lesquels le pape prend des mesures intransigeantes. De surcroît, une analogie s'impose grâce aux propriétés caractéristiques du produit désigné par le $N d M$ en question qui ont un pouvoir catégorisant que l'on pourrait traduire ainsi: des manières totalisantes d'éradication du problème. Ce pouvoir catégorisant traverse les groupes sociaux et les genres textuels.

\subsection{Instantané des issues de l'interaction interculturelle déclenchée par Kärcher}

Dans un premier temps, nous allons montrer que le nom Kärcher qui par antonomase était associé à "racaille" à ses origines, évolue dans les discours et affecte d'autres sphères. À savoir, les scripteurs francophones natifs ou non (les frontières de la blogosphère par définition sont ouvertes comme on vient de le démontrer mais la connotation culturelle de l'extension de domaine nationale semble tout de même primer) s'approprient le $N d M$ Kärcher et le transforment à leur guise pour l'appliquer aux domaines éloignés du sens propre et figuré du terme: juridique, économique, des mœurs, comportemental. Finalement, l'interculturel comme "dynamique entre" échappe à l'aspect apparemment figé du culturel et se manifeste comme une dynamique entre domaines différents.

À la lecture des tableaux 4., 5. et 6., nous nous interrogeons sur le concept de référence en didactique des langues et sa pertinence par rapport à la lexie Kärcher que nous avions définie en introduction comme "un ensemble d'informations qui émergent des données" (Conrad, 2010). Nous nous inscrivons dans la définition que Jean-Claude Milner propose de la "référence", à savoir:

Le segment de réalité associé à une séquence est sa référence actuelle; l'ensemble de conditions caractérisant une unité lexicale est sa référence virtuelle. On voit aisément que cette dernière notion saisit ce qu'on appelle volontiers le sens lexical, et de fait, la référence virtuelle d'une unité est bien ce que tente de représenter la définition du dictionnaire. Simplement, au lieu que cette dernière doive être prise pour une paraphrase ou une traduction ou une représentation verbale (ou même imagée) d'une entité indéfinissable, dite sens, elle obtient un statut clair: des conditions requises à l>égard d`une réalité. (Milner, 1976: 64)

Finalement, nous concluons avec Milner qu'“une unité lexicale ne peut avoir de référence actuelle que si elle est employée" (ibid.). Certes, toutes les néoformations gravitant autour de la lexie Kärcher lorsqu'elles n'interviennent qu'une seule fois ${ }^{12}$ ne constituent point la "référence" lexicale. En revanche, les formes verbales "kärcheriser" ou "karchériser" rem-

12 Cela permet de définir ces néoformations comme un hapax. 
portent au total 44 occurrences, ce qui garantirait une certaine saillance quantitative. Nous rendrons compte de leur représentativité en discours en contexte ${ }^{13}$.

À l'issue de cette fouille, nos bases empiriques comportent respectivement: 181 résultats dans Araneum et 1713 résultats dans frTenTen. Suite à un tamisage manuel, nous avons retenu 21 occurrences issues de Araneum et 71 occurrences relevant de frTenTen.

Du point de vue diachronique, nous avons cerné des occurrences s'échelonnant entre le 15 décembre 2005 et le 3 février 2017 dans frTenTen. En revanche, la proposition d'une datation précise est plus difficile dans Araneum et nous ne sommes pas sans rappel des problèmes d'investigabilité des textes auxquels les discours numérique ${ }^{14}$ nous exposent:

(1) Le chanteur a défendu les propos de Nicolas Sarkozy contre les "racaille" ou la nécessité de "nettoyer au Kärcher" les banlieues, qui avaient contribué à embraser les quartiers à l'automne dernier. (Araneum, denistouret.net, date non disponible)

(2) Nettoyer ces zones de non droit au "kärcher". (Araneum, francaisdefrance.wordpress.com, date non disponible)

(3) L'adhésion des populations éloignées des grands centres de décision (territoires ruraux, ceintures péri-urbaines) et de celles des cités où, kärcher ${ }^{\circledR}$ ou non, la politique sécuritaire est inexistante. (Araneum, sortiedequiescence.blogs.nouvelobs.com, date non disponible)

La propriété référentielle de la pompe à eau hautement nettoyante semble encore solidement liée aux contextes des cités ou des quartiers difficiles en général. Le signe linguistique Kärcher est utilisé comme foyer d'une métaphore à ses origines créative (Prandi, 2016) qui s'est désormais stabilisée dans le discours au quotidien $(1,2)$ mais qui intègre également les discours des blogs de la presse avec la mise en place d'une ellipse dans le contexte (3) au vu de la suppression du verbe "nettoyer" parce que le signe Kärcher fait assez entendre pour qu'il ne reste ni obscurité ni incertitude.

(4) Notons qu'à l'extrême-droite MLP bénéficie elle aussi de cet effet repoussoir après que NS ait siphonné sans vergogne les suffrages des adeptes $d u$ kärcher pour les mettre au service de sa rolex. (Araneum, leblogdekali.blogs.nouvelobs.com, date non disponible)

(5) L'électorat du petit Nicolas pourrait bien se montrer saisi d'une volatilité extraordinaire, faisant par pur dépit franchir à MLP la barrière des $25 \%$ (il a tellement travaillé

13 Nous ne nous attardons pas sur la syntaxe d'interrogation en $C Q L$ (Contextual Query Language) qui a été mise en place et qui est indéniablement fondamentale pour puiser correctement dans les corpus électroniques. À cet effet et pour promouvoir la recherche des collocations d'usage en cours de Langue culture LV1 français 2 année et Médiation de l'IT vers le FR LV2 en 2ème ou 3ème année de Licence, les étudiant.e.s maîtrisent les rudiments de la recherche outillée.

$14 \mathrm{Au}$ vu des nombreuses fautes que les occurrences des corpus frTenTen et Araneum contiennent, nous précisons qu'il s'agit des contextes authentiques et que nous ne les signalons pas. 
Anales de Filología Francesa, n. ${ }^{\circ}$ 29, 2021

POUR UN DIALOGUE INTERCULTUREL: ÉVOLUTION SÉMANTIQUE DU NOM DE MARQUE KÄRCHER...

à désinhiber les manieurs de kärcher ...). (Araneum,leblogdekali.blogs.nouvelobs.com, date non disponible)

(6) Pas de promesse de kärcher; des arguments chocs, tout de suite ... (Araneum, pasidupes.blogspot.fr, date non disponible)

(7) Une racaille qui ne se doute de l'arrivée d'un kärcher humain. (frTenTen, date non disponible)

(8) Si le kärcher policier arrive, nous voulons être de nouveau des milliers pour donner corps aux "Vinci dégage!" et revenir occuper la zone afin de continuer à empêcher concrètement les travaux. (Araneum, zad.nadir.org, Jeudi 28 juin 2012)

La batterie de contextes $(4,5,6)$ affiche de nouvelles collocations où le collocateur Kärcher en association avec le collocataire 15 "adeptes", "promesses", "manieurs" se présentent comme des néoformations à expansion nominale pour désigner les électeurs ayant un positionnement politique plutôt de droite et une personnification du policier qui exprime une propension à la gestion des banlieues, à l'éradication des problèmes de ces dernières, de manière rapide et immédiate (7); la collocation "manieurs de kärcher" (5) serait une variante fantaisiste pour illustrer un travail d'intervention massive de la part des corps de police préconisée par le gouvernement de l'époque; un prolongement de cette personnification est par ailleurs repérable dans le contexte (8) appliquée au domaine de la protestation, des manifestations sociales contre des actions d'urbanisme immodéré aux dires du scripteur qui sollicite le mouvement de protestation tonnant contre une entreprise de Bâtiment et Travaux publics français ("Vinci dégage!").

Nous parvenons à une première migration interculturelle dans ce détournement de lexie recensé par l'énoncé (8), du domaine politique prioritairement lié aux banlieues au domaine social.

Domaine de l'urbanisme:

(9) Et sur le dossier sdf, il avait fait l'impasse, l'ancien balladurien. Il ${ }^{16}$ ne va pas kärcheriser les trottoirs. (frTenTen, http://filaplomb.over-blog.org/article-4998725.html, 23 décembre 2006)

(10) M. Jean-Claude Gaudin et son adjoint à l'urbanisme (Marseille), qui rénovent au kärcher: "On a besoin de gens qui créent de la richesse". (frTenTen, https://www. monde-diplomatique.fr/2007/01/ruffin/14324, janvier 2007)

15 Nous empruntons la terminologie à GRÉCIANO, Gertrud. 1997. "Collocations rythmologiques" in Meta, $\mathrm{n}^{\circ} 42$, 33-44.

16 Il est utile d'observer l'allusion à l'ancien balladurien N. Sarkozy et à sa campagne présidentielle menée lors des élections de 2007. 
L'apparition du phraséologisme (10) "rénover au Karcher" pour illustrer une action d'intervention expéditive d'aménagement urbain à Marseille dénote une évolution de l'implantation de la lexie et de ses dérivés dans d'autres sphères comme l'urbanisme. La tournure "rénover au kärcher" désigne une action d'intervention qui n'est pas forcément à l'avantage du centre-ville tout comme la méthode d'assainissement préconisée en 2005 par son Ministre de l'Intérieur ne l'était pas davantage. L'évolution est éclairante du point de vue conceptuel - nous semble-t-il - car la "méthode Karcher" (sic) dépasse les frontières des banlieues et deviendrait une réalité partagée par la communauté aussi bien banlieusarde que citadine. L'introduction du dénominal "kärcheriser" est exploitée (9) comme substitut plus efficace de nettoyer les trottoirs et bien dans d'autres contextes assez hétéroclites où la datation temporelle est éclairante. Nous allons les analyser en contexte.

Domaines des idées, de l'environnement, de l'Education nationale et du droit:

(11) $\mathrm{Il}^{17}$ a donc promis de "kärcheriser" (ce mot-là, il me fait mal, mais tant pis) non pas les idées -ce serait mal le connaitre- mais la vulgarité. (frTenTen, http://www.maitreeolas.fr/post/2005/12/15/250-nos-amis-les-facheux, 15 décembre 2005)

(12) "kärcheriser" ce qui a été fait en matière d'éducation nationale au cours de la précédente législature. (frTenTen, assemblee-nationale.fr, 17 janvier 2013)

(13) J'ai entendu çà et là qu'il suffirait aux entreprises de pratiquer l'hydrofracturation sans le dire - notre collègue Yves Cochet a parlé de "kärcher souterrain". Grâce à la disposition introduite par cet amendement, ce ne sera pas possible, car ils seront soumis au contrôle des acteurs locaux, dont la mobilisation ces derniers mois a montré la détermination à empêcher la dénaturation de leurs territoires. (frTenTen, Assemblée nationale $\sim$ Première séance du mardi 21 juin 2011)

(14) Le Grenelle au kärcher. (frTenTen, eauxglacees.com 30 octobre 2007)

(15) Qu'est-ce qu'“un recteur kärcherisé"? Tout simplement un haut fonctionnaire qui, un jour, se voit limogé en Conseil des ministres alors qu'il croyait défendre la laïcité de l'enseignement. (frTenTen, nouvelobs.com, 22 mars 2007)

(16) Depuis maintenant cinq années, il est clair que le système financier et nos dirigeants qui lui servent de paravents, mènent à la destruction des peuples et des nations. Le salut passe inévitablement - une fois les banques passées au kärcher - par l>emploi des technologies et des sources d`énergie d`avenir. (frTenTen, solidariteetprogres.org, 30 novembre 2013)

(17) Les adversaires du Cal Ratzinger l'appelaient le Panzerkardinal. Ce qui est sûr, c'est que c'est le Kärcherpabst, plus efficace que Sarkozy en banlieue: tolérance zéro, il bat des records de limogeages. (frTenTen, plunkett.hautetfort.com, 24 juin 2012)

17 Au vu de l'opacité de l'énoncé, nous ne parvenons pas à identifier son énonciateur. 
Avançant dans notre fouille, nous nous apercevons que les scripteurs ont toute latitude (cf. (13) et (14)) pour décrire par un mot évènement la mise à mal d'une série de décisions prises à long terme en matière d'environnement et de développement durable aux dires du blogueur le 30 octobre 2007, alors qu'en (13), dans une séance de l'Assemblée nationale une technique d'extraction permettant, entre autres, d'extraire des hydrocarbures est dénommée comme un "Karcher souterrain". Le raccourci métonymique est ici mis en place en exploitant la relation suffisamment nette entre l'aspirateur à ses origines et cette technique d'extraction aux impacts environnementaux controversés. L'occurrence (12), également tirée lors d'une séance de l'Assemblée Nationale, montre que les sèmes véhiculés par le signe linguistique Kärcher et ses néoformations affectent des domaines sociétaux de plus en plus diversifiés, et à tous les niveaux de la société.

L'analogie intrinsèque basée sur les effets de l'événement initial de 2005 est repérable dans les contextes (15), (16) et (17) où le pouvoir catégorisant de l'analogie semble inférer un sens supplémentaire au phraséologisme: "passer au kärcher [les banques]" (16), à la composition "Kärcherpabst" (17) et au groupe nominal "un recteur kärcherisé" (15), à savoir celui de limogeage spectaculaire. Finalement, les énoncés (15), (16) et (17) montrent que "les transferts, les branchements interculturels [de la lexie kärcher] et [sa] circulation dans les médias sont cruciaux pour constituer des 'sas' de reconnaissance et d'évolution des appartenances socio-économiques, à tous les niveaux de chaque société et entre les sociétés", comme nous l'apprend Demorgon (2003). De la "racaille" aux Recteurs d université, aux Présidents d'établissements bancaires et aux hauts prélats, la dérivation "karcheriser" sert, au fil du temps, à désigner le licenciement de représentants des plus hautes sphères de la hiérarchie sociale. Serait-il possible de voir là une sorte de migration interculturelle et intergroupale? Il nous semble que la riche palette de contextes le montre.

En rappel du concept d'interculturel factuel de notre époque que Demorgon appelle "l'informationnel-mondial" (2003), les contextes d'usage de la lexie Kärcher nous apprennent que les acteurs utilisent les cultures des autres dans un processus d'accommodation de soi aux réalités et d'assimilation des réalités à leurs données propres:

(18) En 2014, la police met à jour une vaste affaire de transferts de fonds, à partir d'une station de lavage de voitures... d'où le nom de l'affaire qui éclabousse le Brésil, "lavajato" (synonyme de Kärcher). (frTenTen, francetvinfo.fr, 31/05/2016)

Alors qu'une traduction mot-à-mot: "lavage jet" pourrait tout à fait convenir en français, le scripteur prévoit une traduction qui n'est point innocente pour la connotation polémique que la lexie Kärcher recèle. 


\subsection{Migrations nouvelles dans l'emploi de la lexie Kärcher}

Nous souhaitons valoriser la recherche documentaire que permettent les fouilles outillées sur corpus avec un panel d'emplois nouveaux recensés notamment dans frTenTen qui montre en perspective interculturelle et par approche diachronique, l'enrichissement des champs sémantiques et des activités humaines affectés par la lexie Kärcher.

(19) Ma maman a dû se passer au Kärcher après sa course! (frTenTen, blog.peinture-etillustration.fr, date non disponible)

(20) Un Kärcher bien puissant. (frTenTen, triathlonspirit.com, date non disponible)

(21) Copies nettoyées au Kärcher. (frTenTen, ahbon.free.fr, date non disponible)

(22) Une check-list où tu coches/rayes/passes au kärcher ce que tu as fait est aussi une bonne façon de rester motivé. (frTenTen, Noël 2015)

(23) Des textes crûs kärcherisant les enceintes au milieu du tumulte musical. (frTenTen, date non disponible)

(24) On peut faire 5 sushis par œuf sans problème. Oui oui, avec un brumisateur vendu en pharmacie ou parapharmacie (pas avec un pulvérisateur, n'exagérez pas, ni avec un kärcher). (frTenTen, culinotests.fr, 29 juillet 2016)

(25) Un effet Kärcher sur l'épiderme. (frTenTen, vogue.fr, 3 février 2017)

De nombreux domaines sont convoqués: de la vie privée qui comporte la prise d'une douche $(19,20)$ à la préparation d'un plat $(24)$, en passant par un soin de la peau (25), l'allégement d'une liste de contraintes au quotidien que l'on enraye au fur et à mesure (22), ou le domaine ludique de la musique et du cinéma $(23,21)$. La lexie Kärcher et ses dérivés semblent bien correspondre au champ notionnel de l'interculturel annoncé par Demorgon (2003). Ils fonctionnent comme un sas de reconnaissance fort riche qui traverse aussi bien une large palette de domaines de l'activité humaine que les différentes catégories d'acteurs qui la composent entraînant leur "société singulière" à se maintenir ou à se modifier.

\section{Conclusion}

Nous inscrivant dans le cadre théorique de Demorgon qui préconise que tout domaine de diversité est susceptible d'une prise en compte interculturelle: couches socio-économiques, professions, discours politique, nous avons démontré que dans un intervalle de temps allant du 15 décembre 2005 au 3 février 2017, la lexie Kärcher s'est enrichie d'une constellation de sèmes en discours. Le sens de ces lexies dépassent largement les frontières de l'événement déclencheur à ses origines. Appréhendant l'interculturel dans son versant 
inter-, tel que Lemoine (2018) le conçoit, nous avons montré la dynamique qui s'enclenche à partir de notre unité lexicale de prédilection. On est en droit, suite à cette brève étude de cas, de considérer que l'unité simple Kärcher et ses dérivés fonctionnent comme puissants activateurs lexiculturels, référence lexicale et référent discursif aussi bien dans les discours de la blogosphère, de la presse que de l'Assemblée Nationale qui circulent sous l'égide d'une zone de nommage en ".fr", marquage d'une identité culturelle certaine. Une plus large diachronie sera profitable pour étudier l'étendue de la contamination de l'unité lexicale Kärcher qui semble avoir récemment intégré le stock lexical des locuteurs pour désigner des activités du quotidien et contribuer peut-être à leur pérennisation. Les réseaux sociaux pourraient constituer de valables ressources auxquelles puiser pour mener une veille lexicale et phraséologique qui permettrait de mettre à jour les références d'usage de Kärcher et de ses dérivés, intégrant ainsi les contextes certes précieux mais cristallisés à la date de conception du corpus respectif que nous avons utilisé.

\section{Références bibliographiques}

Araneum Francogallicum Maius: <https://kontext.korpus.cz/> [21/05/2021].

Boulton, Alex \& Henry Tyne. 2014. Des documents authentiques aux corpus: démarches pour l'apprentissage des langues. Paris, Didier (coll. Langues et didactique).

Cislaru, Georgeta. 2013. "Pratiques langagières de 1"événementialisation': illustrations dans le discours médiatique" in Ballardini, Elio, Pederzoli, Roberta, Reboul-Touré, Sandrine \& Geneviève Tréguer-Felten (éds.). Les facettes de l'événement: des formes aux signes, mediAzioni, $\mathrm{n}^{\circ} 15:<\mathrm{http}: / /$ mediazioni.sitlec.unibo.it> [4/03/2021].

Demorgon, Jacques. 2003. "L'interculturel entre réception et invention. Contextes, médias, concepts" in Questions de communication, $\mathrm{n}^{\circ} 4:<\mathrm{http}$ ://journals.openedition.org/questionsdecommunication/4538> [05/03/2021].

French Web 2017 (frTenTen17): <https://app.sketchengine.eu/\#dashboard?corpname=preloa ded\%2Ffrtenten17_fl2> [07/03/2021].

Galisson, Robert. 1991. De la langue à la culture par les mots. Paris, Clé-International.

Galisson, Robert \& Andre, Jean-Claude. 1998. Dictionnaire de noms de marques courants: essai de lexiculture ordinaire. Paris, Didier Érudition.

Lemoine, Véronique. 2018. "L'interculturel en réflexion pour la classe et ailleurs" in Recherches en didactiques, $\mathrm{n}^{\circ} 25,77-92$.

Milner, Jean-Claude. 1976. "Réflexions sur la référence” in Langue française, n 30, 63-73.

Neveu, Erik \& QuéRÉ, Louis (éds.). 1996. “Le temps de l'événement 1" in Réseaux, № 75, 7-21 et "Le temps de l'événement 2" in Réseaux, n 76, 5-7.

Paveau, Marie-Anne. 2017. Analyse du discours numérique: dictionnaire des formes et des pratiques. Paris, Hermann. 
Prandi, Michele. 2016. "L'interaction métaphorique: une grandeur algébrique" in Protée, numéro thématique Le groupe $\mu$ entre rhétorique et sémiotique: archéologies et perspectives, vol. $38, \mathrm{n}^{\circ} 1,75-84$.

Ricoeur, Pierre. 1991. "Evénement et sens” in Raisons pratiques, n², 41-56.

Sablayrolles, Jean-François \& Pruvost, Jean. 2016. Les néologismes. Paris, PUF (coll. Que sais-je?).

Sinclair, John. 2004. "New evidence, new priorities, new attitudes" in SinclaIR, John (éd.), How to use corpora in language teaching. Amsterdam, John Benjamins Publishing Company, 271-299.

SREDANOVIC, Djordje. 2014. "Les industries culturelles et la permanence du national à l'ère d'Internet", Les Enjeux de l'Information et de la Communication, n¹5/2a, 67-79. <https:// lesenjeux.univ-grenoble-alpes.fr/2014/supplement-a/06-les-industries-culturelles-et-la-permanence-du-national-a-lere-dinternet> [4/04/2021].

TonTI, Michela. 2019. Lexiculture et linguistique: une approche guidée sur corpus, des noms de marque dans le discours au quotidien. Thèse de doctorat. Alma Mater Studiorum Università di Bologna.

TonTI, Michela. 2020. Le nom de marque dans le discours au quotidien: prisme lexiculturel et linguistique. Paris, L'Harmattan (coll. Laboratorio@francesisti.it).

AFNIC. Association française pour le nommage Internet en coopération: <www.afnic.fr> [21/03/2021]. 
